\title{
Development and validation of a prediction index for recent mortality in advanced COPD patients
}

\author{
Sheng-Han Tsai ${ }^{1}$, Chia-Yin Shih ${ }^{2}$, Chin-Wei Kuo ${ }^{3}$, Xin-Min Liao ${ }^{3}$, Peng-Chan Lin ${ }^{4}{ }^{4}$, Chian-Wei Chen ${ }^{3}$, Tzuen-Ren Hsiue ${ }^{3,5}$ and \\ Chiung-Zuei Chen (iD ${ }^{3,5} \bowtie$
}

The primary barrier to initiating palliative care for advanced COPD patients is the unpredictable course of the disease. We enroll 752 COPD patients into the study and validate the prediction tools for 1-year mortality using the current guidelines for palliative care. We also develop a composite prediction index for 1-year mortality and validate it in another cohort of 342 patients. Using the current prognostic models for recent mortality in palliative care, the best area under the curve (AUC) for predicting mortality is 0.68 . Using the Modified Medical Research Council dyspnea score and oxygen saturation to define the combined dyspnea and oxygenation (DO) index, we find that the AUC of the DO index is 0.84 for predicting mortality in the validated cohort. Predictions of 1 -year mortality based on the current palliative care guideline for COPD patients are poor. The DO index exhibits better predictive ability than other models in the study.

npj Primary Care Respiratory Medicine (2022)32:2 ; https://doi.org/10.1038/s41533-021-00263-7

\section{INTRODUCTION}

The prevalence of and mortality associated with chronic obstructive pulmonary disease (COPD) have been increasing annually ${ }^{1}$. However, current treatments have been disappointing in terms of controlling airflow obstructions and reducing mortality ${ }^{2-4}$. Although palliative care is shown to be effective in patients with COPD, these patients have fewer opportunities to receive palliative care than patients with cancer $^{5,6}$. Jabbarian et al. found that the failure to implement advance care planning (ACP) in chronic diseases is mainly due to the complexity and unpredictability of the disease ${ }^{7}$, and the uncertainty of disease trajectory is even greater in COPD than in cancer ${ }^{8-12}$. In addition, COPD patients typically want to know more about their prognosis in the early stages ${ }^{13,14}$. Therefore, enormous effort has been made to find indicators to predict a poor prognosis accurately.

Researchers have found many indicators related to various adverse outcomes for COPD, including patient age, body mass index (BMI), dyspnea, smoking status, exercise capacity, acute exacerbation, symptoms, and biological indicators ${ }^{15-17}$. Unfortunately, as was the case with the first proposed indicator, FEV 1 , there was no optimal way to predict mortality based on the indicator ${ }^{17,18}$. After the multisystem involvement characteristic of COPD became known, the focus was moved to composite indicators to achieve better predictive outcomes ${ }^{15,19}$. The earliest developed and most widely investigated multicomponent indicators included the Body-Mass Index, Airflow Obstruction, Dyspnea, and Exercise Capacity (BODE) Index ${ }^{20}$, which was also recommended for predicting outcomes by the Global Initiative for Chronic Obstructive Lung Disease (GOLD) ${ }^{21}$. Later, numerous different indices were developed, including the Dyspnea and Airflow Obstruction (ADO) Index, the Dyspnea, Obstruction, Smoking, Exacerbation (DOSE) Index, and various modifications of the BODE index ${ }^{22}$. However, most of these indices were developed to predict long-term survival. They all lacked accuracy when applied to short-term events of $<12$ months ${ }^{20-23}$. Marin et al. ${ }^{23}$ validated a number of existing prognostic indices in a large individual pooled data set $(n=3633)$ from multiple cohort studies with different stages of COPD. These prognostic indices included the original BODE, the modified BODE (replacing the 6-min walk distance (6MWD) with peak oxygen uptake $\mathrm{V}_{\mathrm{O} 2}$ as $\%$ predicted), the BODEx (replacing the 6MWD with exacerbations), the eBODE (BODE plus exacerbations), the SAFE (SGRQ score, air-flow limitation, and exercise tolerance), the ADO, and the DOSE. Allcause mortality prediction at 12 months was assessed for these indices, where the indices determined to be optimal for prediction was the ADO ( $C$ statistic $=0.70)$. Boeck et al..$^{24}$ developed the B-AE$\mathrm{D}$ indices (BMI, acute exacerbations, dyspnea) for 2-year mortality in the PROMISE study, and external validation of the B-AE-D was performed in COCOMICS and the COMIC study for 1-year all-cause mortality ( $C$ statistic $=0.68$ and 0.74 , respectively). Therefore, none of these indices had the strong predictive ability for 1-year mortality. In addition, none of these models were developed with the specific aim of predicting all-cause mortality in stable COPD patients within 12 months.

To the best of our knowledge, Bloom et al. ${ }^{25}$ was the only research group to develop indicators (the BARC index) for predicting 1-year mortality with the aim of palliative care in advanced COPD ( $C$ statistic $=0.78$ and 0.70 for the development and validation cohorts, respectively). The variables in the BARC only required routinely collected non-specialist information, which, therefore, helped identify patients seen in primary care institutions, but a total of 18 variables were required. Because no existing indices had strong enough predictive ability for 1-year mortality in clinical practice, and very few indices were developed with the specific aim of predicting 1-year mortality for palliative care in stable COPD. In this study, we aimed to validate the currently recommended prediction indices for palliative care, we

\footnotetext{
${ }^{1}$ Division of General Medicine, Department of Internal Medicine, National Cheng Kung University Hospital, College of Medicine, National Cheng Kung University, Tainan, Taiwan. ${ }^{2}$ Department of Public Health, College of Medicine, National Cheng Kung University, Tainan, Taiwan. ${ }^{3}$ Division of Pulmonary Medicine, Department of Internal Medicine, National

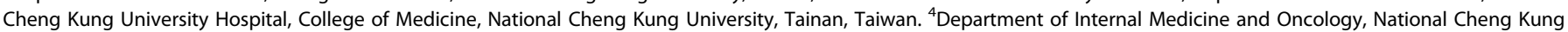
University Hospital, College of Medicine, National Cheng Kung University, Tainan, Taiwan. ${ }^{5}$ These authors contributed equally: Tzuen-Ren Hsiue, Chiung-Zuei Chen.

email: chen96@mail.ncku.edu.tw
} 
also developed a new predictive index for 1-year mortality in hospitalized ambulatory COPD patients.

\section{METHODS \\ Study design}

We conducted this cohort study in the National Cheng Kung University Hospital (NCKUH) from August 2006 to December 2015. The patients included in the present study were part of another previous study ${ }^{26}$. The patients were eligible for inclusion if they had received regular management for COPD at our hospital for $>1$ year prior to their recruitment. All patients were diagnosed with COPD by pulmonologists according to the GOLD guidelines for diagnostic criteria ${ }^{1}$. The criteria were as follows: age $>40$ years, typical symptoms, such as cough, dyspnea, wheezing, or chest tightness in combination with evidence of chronic airflow obstruction, as defined by a postbronchodilator ratio of forced expiratory volume in $1 \mathrm{~s}\left(\mathrm{FEV}_{1}\right)$ to a forced vital capacity (FVC) of $<70 \%$. Pulmonary function tests were performed following the standard protocols of the American Thoracic Society ${ }^{27}$. All patients were enrolled under clinically stable conditions. We excluded patients who were unwilling to participate and those who had advanced lung cancer and pulmonary fibrosis because of anticipated death in the near future. Patients with missing data and those lost to follow-up in the first year were also excluded from the analysis. In total, 752 patients with COPD were analyzed (Supplementary Fig. 1). The Institutional Review Board of NCKUH approved this study before commencement (IRB number: B-ER105-386 and B-ER-98-289). Written informed consent was obtained for all participants while enrollment.

\section{Prognostic variables and outcome}

A total of 752 consecutive COPD patients were recruited. All patients were monitored through December 2016 or until death. We acquired age, smoking history, BMl, the severity of dyspnea assessed by grade on the modified Medical Research Council (mMRC) dyspnea scale ${ }^{28}$, the degree of comorbidity as evaluated using the Charlson index ${ }^{29}$, oxygen saturation levels as detected by pulse oximetry in room air $\left(\mathrm{SpO}_{2}\right)$, and status of long-term home oxygen usage from every patient at the time of inclusion as determined by research assistants in the study. Comorbidity was evaluated using the Charlson index and included congestive heart failure, coronary artery disease, systemic hypertension, peptic ulcer, and diabetes mellitus as identified from the patient files and detailed interviews. A severe acute exacerbation of COPD was defined as an acute event characterized by a worsening of the patient's respiratory symptoms that were beyond day-to-day variations that also required hospitalization. The number of severe exacerbations in the preceding year was recorded by research assistants according to the patient's chart as the primary means of data collection; self-reported data was used to supplement this data.

All-cause mortality was defined as the endpoint of the study. The survival status of all patients was evaluated using a prospective observation, as reported in a previous study ${ }^{26}$. All patients were contacted during regular clinic visits or by telephone interviews (if they missed an appointment). Most patients who died during the study period had been regularly followed and had visited the hospital for treatment before their death. Their dates of death were recorded and verified using hospital records. Research assistants obtained the date of death of patients who died outside the hospital by telephone contact with partners or family members. Survival status was also verified through linkage with the Taiwan National Mortality Registry.

\section{Predictive variables for palliative care}

In the first part of the study, we evaluated the predictive ability of the currently recommended variables for estimating 1-year mortality in the palliative care guideline for COPD. We selected several variables for building the predictive model based on a review of the currently recommended prediction variables ${ }^{30-32}$. The variables included (1) $\mathrm{mMRC}$ score $=4$, (2) frequent, severe $A E$ (two or more AEs requiring hospitalization in the preceding year), (3) hypoxemia $\left(\mathrm{SpO}_{2}<90 \%\right.$ in ambient air), (4) $\mathrm{BMI}<21$, and (5) predicted $\mathrm{FEV}_{1}<30 \%$. We used several combined indices to test the accuracy of the prediction for 1-year mortality. The patients were subdivided into four groups: Group 1 was defined as patients with frequent, severe $\mathrm{AE}$ in combination with severe dyspnea $(\mathrm{mMRC}=4)$. Group 2 was defined as patients with frequent, severe $\mathrm{AE}$ in combination with $\mathrm{SpO}_{2}<90 \%$ in ambient air. Group 3 was defined as patients with frequent, severe $A E$ combined with predicted $\mathrm{FEV}_{1}<30 \%$. Group 4 was defined as patients with frequent, severe $A E$ in combination with $B M I<21$.

\section{Modeling the predictive scores}

Because of the generally unsatisfactory predictive power found in previous studies and with validating our results, we wanted to derive a new predictive model for 1-year mortality from the patient variables, including age, sex, BMI, disease severity, such as mMRC dyspnea score, $\mathrm{FEV}_{1}, \mathrm{SpO}_{2}$, and comorbidities. The variables were evaluated using multivariate Cox regression models with a forward entering approach and a 5\% significance level for the selection criteria. Significant regression coefficients were converted to exponential expressions for the weighting of the variables used for the predictive indices.

\section{Validation of the predicting index}

To validate the predictive performance of our model, we selected a second cohort. All patients in the development group were recruited from pulmonary outpatient departments. Considering that if the validation group and the developmental group exhibited high homogeneity, it was expected that the proposed model would obtain very similar results for the two groups of patients. Patients in the validation group were recruited by screening individuals who had been diagnosed with COPD, not only in the pulmonary outpatient department but also in the Center for Hospice Palliative Shared Care at NCKUH from July 2012 to August 2019. All patients were aged $\geq 40$ years; COPD was defined according to the GOLD diagnostic guidelines and criteria as the developmental group; patients with advanced lung cancer or pulmonary fibrosis were excluded. The date of recruitment of some patients from the Center for Hospice Palliative Shared Care overlapped with the time periods during which the development group was recruited. These patients were not excluded from this study since the source of patients was different from that for the development group (Center for Hospice Palliative Shared Care versus the pulmonary outpatient department). All patients had complete follow-up for 1 year or until death.

\section{Statistical analysis}

Continuous variables are presented as the median and interquartile range because the number of deaths was not large and therefore may not follow a normal distribution. Therefore, comparisons between survivors and nonsurvivors were performed using Mann-Whitney U-test. Comparisons between categorical variables were performed using chi-square tests or Fisher's exact tests.

Kaplan-Meier survival curves and log-rank tests were used for comparing different predictive variables. The ability to predict mortality within 1 year was analyzed using logistic regression models and the receiver operating characteristic (ROC) curve to 
calculate the area under the curve (AUC). Data processing and analyses were performed using the SPSS for Windows version 17.0 statistical software (IBM, Armonk, NY, USA).

\section{Reporting summary}

Further information on research design is available in the Nature Research Reporting Summary linked to this article.

\section{RESULTS}

\section{Participants}

We enrolled 752 COPD patients from August 2006 through December 2015. The mean age of the patients was 70.6 years, and most of them were men (92.7\%). Twenty-eight percent of the patients had severe to very severe airflow limitations $\left(\mathrm{FEV}_{1}<50 \%\right.$ of predicted); $25.2 \%$ of the patients had mMRC scores from 3 to 4 , and $50.7 \%$ had at least one severe $A E$ in the preceding year during enrollment. At the end of the follow-up period in December 2016, 378 patients had died (50.3\%), and 60 patients (8\%) had died within 1 year after the start of follow-up.

The baseline characteristics of survivors and nonsurvivors are shown in Table 1. Compared with survivors, nonsurvivors were older, had a worse pulmonary function, lower BMI, lower oxygen saturation, worse symptoms of dyspnea (higher mMRC score), and more AEs in the previous year.

\section{Predictive ability of the currently recommended models}

The AUC values for predicting 1-year mortality in patients with severe dyspnea $(\mathrm{mMRC}=4)$ and patients having frequent, severe $\mathrm{AE}$ were 0.62 and 0.60 , respectively. Combining predictor variables for patients with severe dyspnea and frequent severe $A E$ was better than using only one variable $(A U C=0.68$ ). The AUC for predicting 1-year mortality for patients with frequent, severe $A E$ and $\mathrm{SpO}_{2}<90 \%$ was 0.66 . The AUC for patients with frequent,

Table 1. Demographic and patient characteristics of survivors and nonsurvivors.

\begin{tabular}{|c|c|c|c|}
\hline Characteristic $^{a}$ & $\begin{array}{l}\text { Survivors } \\
(n=692)\end{array}$ & $\begin{array}{l}\text { Nonsurvivors } \\
(n=60)\end{array}$ & $p$ Value \\
\hline Age, median (IQR) & $71.2(64.6,78.7)$ & $78.4(72.5,81.6)$ & $<0.01$ \\
\hline Male $n(\%)$ & $640(92.5)$ & $57(95.0)$ & 0.61 \\
\hline $\begin{array}{l}\text { Current smoker, } \\
n(\%)\end{array}$ & $189(27.3)$ & $13(21.7)$ & 0.30 \\
\hline $\begin{array}{l}\text { Smoking quantity } \\
\text { (pack-years) }\end{array}$ & $45(23,70)$ & $50(20,62)$ & 0.92 \\
\hline $\mathrm{FEV}_{1} \%$ & $64(48,82)$ & $50(34,64)$ & $<0.01$ \\
\hline BMI & $23.3(20.5,25.8)$ & $20.5(17.0,24.5)$ & $<0.01$ \\
\hline $\mathrm{SpO}_{2} \%$ & $97.0(95.0,98.0)$ & $95.5(92.0,97.0)$ & $<0.01$ \\
\hline $\mathrm{Cl}$ score & $2.0(1.0,3.0)$ & $3.0(1.0,5.0)$ & $<0.01$ \\
\hline Severe $A E \geq 2, n(\%)$ & $111(16.0)$ & $22(36.7)$ & $<0.01$ \\
\hline 6MWT (meter) & $344.0(248.0,400.0)$ & $278.0(206.0,313.0)$ & 0.11 \\
\hline SGRQ score & $33.22(18.2,51.0)$ & $59.13(46.4,65.3)$ & $<0.01$ \\
\hline $\mathrm{mMRC}=4$ & $26(3.7)$ & $17(28.3)$ & $<0.01$ \\
\hline LTOT, $n$ (\%) & $71(10.3)$ & $15(25.0)$ & $<0.01$ \\
\hline
\end{tabular}

${ }^{\text {aD }}$ iscrete data are presented as number (percentage), and continuous variables are presented as median (IRQ).

$\mathrm{FEV}_{1}$ forced expiratory volume in $1 \mathrm{~s}, \mathrm{BMI}$ body mass index, $\mathrm{SpO}_{2}$ oxygen saturation (\%) detected with pulse oximeter when breathing in room air, $\mathrm{Cl}$ Charlson index, severe $A E \geq 2$ history more than one acute exacerbation that required hospitalization in the preceding year, $6 M W T 6 \mathrm{~min}$ walking test, SGRQ St. George's Respiratory Questionnaire, $m M R C$ modified Medical Research Council Dyspnea Scale, LTOT long-term oxygen therapy. severe $\mathrm{AE}$ and predicted $\mathrm{FEV}_{1}<30 \%$ was 0.60 , and the $\mathrm{AUC}$ for patients with frequent, severe $A E$ and $B M I<21$ was 0.68 . The ROC for the different composite indices did not differ significantly between the four groups of combinations (Fig. 1).

Kaplan-Meier survival analysis for all-cause mortality showed the 1-year survival rates for groups 1-4 were $62,75,88$, and $78 \%$, respectively (Fig. 2). All indices, including those for groups 1-4, showed high specificity but unsatisfactory sensitivity. The composite indices for group 1 with frequent, severe $A E$ and severe dyspnea had a better positive predictive value than the other groups (Table 2).

\section{Development and validation of a prediction index}

In the univariate analysis, there were significant differences between survivors and nonsurvivors in age, $\mathrm{FEV}_{1}, \mathrm{SpO}_{2}, \mathrm{mMRC}$, $\mathrm{BMI}$, anemia, and concurrent malignancies. Using multivariate regression, only $\mathrm{mMRC}$ and $\mathrm{SpO}_{2}$ were independent risk factors for predicting 1-year mortality.

We refined the dyspnea and oxygenation (DO) index by weighting dyspnea and $\mathrm{SpO}_{2}$ based on the results of the multivariate regression model. We used the integers closest to the hazard ratio for scoring predictive variables (Table 3). Compared with groups 1-4, the DO index had better discrimination for mortality (AUC $=0.73$; Fig. 1). We also performed a sensitivity analysis for patients with severe or very severe obstruction $\left(\mathrm{FEV}_{1}<50 \%\right)$, where the $\mathrm{DO}$ index performed better $(A \cup C=0.81)$. The survival rates for the different DO scores are

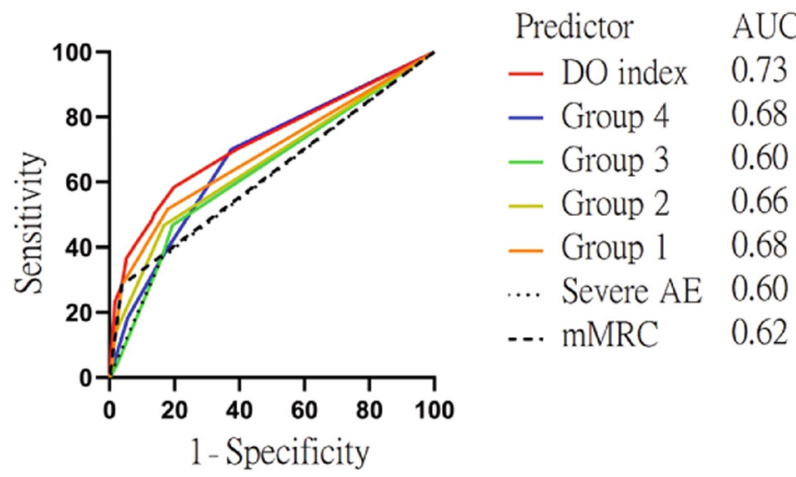

Fig. 1 ROC curve for severe dyspnea, severe acute exacerbation, and different combinations of predictors and DO index for 1-year mortality in COPD patients. The AUC values for patients with severe dyspnea $(m M R C=4)$, frequent severe $A E$, groups $1-4$, and $D O$ index were $0.62,0.60,0.68,0.66,0.60,0.68$, and 0.73 , respectively.

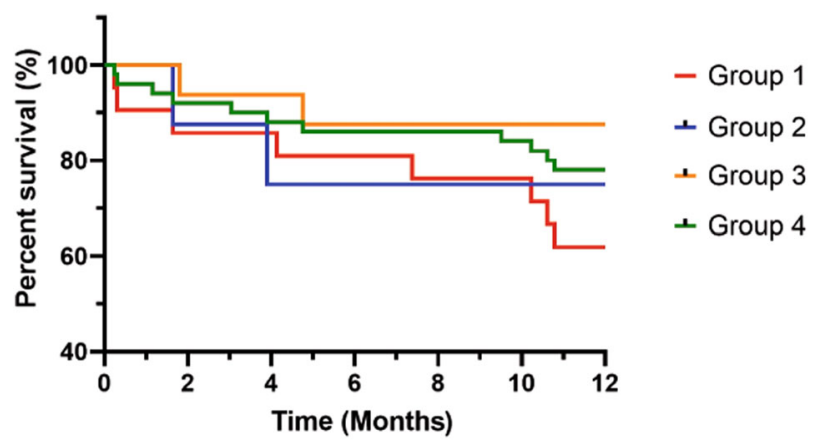

Fig. 2 Kaplan-Meier survival curves for 1-year mortality according to different recommended prediction indices for palliative care. The 1-year survival rates were $62,75,88$, and $78 \%$ for groups $1-4$, respectively. 
Table 2. Predictive accuracy of different recommended palliative care indices for 1-year mortality.

\begin{tabular}{|c|c|c|c|c|c|c|}
\hline Prognostic index & Sensitivity & Specificity & PPV & NPV & Accuracy & AUC \\
\hline $\mathrm{mMRC}=4$ & $28.3 \%$ & $96.2 \%$ & $39.5 \%$ & $93.9 \%$ & $90.8 \%$ & 0.623 \\
\hline Severe $A E \geq 2$ & $36.7 \%$ & $84.0 \%$ & $16.5 \%$ & $93.9 \%$ & $80.2 \%$ & 0.603 \\
\hline Group 1 & $13.3 \%$ & $98.1 \%$ & $38.1 \%$ & $92.9 \%$ & $91.4 \%$ & 0.684 \\
\hline Group 2 & $3.3 \%$ & $99.1 \%$ & $25.0 \%$ & $92.2 \%$ & $91.5 \%$ & 0.657 \\
\hline Group 3 & $3.3 \%$ & $98.0 \%$ & $12.5 \%$ & $92.1 \%$ & $90.4 \%$ & 0.634 \\
\hline Group 4 & $18.3 \%$ & $94.4 \%$ & $22.0 \%$ & $93.0 \%$ & $88.9 \%$ & 0.679 \\
\hline
\end{tabular}

$m M R C$ modified Medical Research Council Dyspnea Scale in stable condition, Severe $A E \geq 2$ more than one acute exacerbation that required hospitalization in the preceding year, Group $1 \mathrm{mMRC}=4+$ severe $\mathrm{AE} \geq 2$, Group 2 severe $\mathrm{AE} \geq 2+\mathrm{SpO}_{2}<90 \%$, Group 3 severe AE $\geq 2+\mathrm{FEV}_{1}<30 \%$, Group 4 severe AE $\geq 2+\mathrm{BMI}$ $<21$, PPV positive predictive value, $N P V$ negative predictive value, $A U C$ area under the curve, $\mathrm{SpO}_{2}$ oxygen saturation (\%) detected with a pulse oximeter when breathing room air, FEV forced expiratory volume in $1 \mathrm{~s}, B M I$ body mass index.

\begin{tabular}{|c|c|c|c|}
\hline Variable & $\beta$ & Adjusted HR & Score \\
\hline \multicolumn{4}{|l|}{$\mathrm{SpO}_{2}(\%)$} \\
\hline $95-100$ & 0 & 1 & 1 \\
\hline $90-94$ & 0.55 & 1.7 & 2 \\
\hline $85-89$ & 1.05 & 2.9 & 3 \\
\hline$<85$ & 2.00 & 7.3 & 7 \\
\hline \multicolumn{4}{|c|}{ mMRC score } \\
\hline $0-2$ & 0 & 1 & 1 \\
\hline 3 & 0.64 & 1.9 & 2 \\
\hline 4 & 2.21 & 9.1 & 9 \\
\hline
\end{tabular}

shown in Table 4. In the group with the most severe COPD (DO score $=12-16$ ), the survival rate was only $20 \%$.

We enrolled a total of 342 patients for the validation group. The patients in the validation group were older (73.5 vs. $72.2 \%)$, had lower oxygenation (96 vs. 97\%), more comorbidities, as evaluated by the $\mathrm{Cl}$ score (4.0 vs. $2.0 \%$ ), more symptoms, as evaluated by the percentage of patients with $\mathrm{mMRC}=4(14.3 \mathrm{vs} .6 .3 \%)$, and higher 1 -year mortality (14.3 vs. $8.0 \%)$, than the developmental group (Supplementary Table 1). When applying our DO score for predicting 1-year mortality, the AUC was 0.84 . In the group with the most severe COPD (DO score $=12-16$ ), the positive and negative predictive values were $87 \%$ and $89 \%$, respectively, for 1-year mortality.

\section{DISCUSSION}

This study showed that a combination of mMRC and frequent, severe $A E$ as a predictor of 1-year mortality demonstrated similar poor discrimination power as other combinations of factors. These factors included predictors with desaturation, or a poor grade of lung function, or low BMl combined with frequent, severe $A E$. The AUC values of these combinations ranged from 0.60 to 0.68 . In addition, all combined groups exhibited lower sensitivity but higher specificity. Therefore, these indices did a good job of ruling outpatients who would survive for $>1$ year but tended to miss patients who would die within 1 year.

Using the mMRC dyspnea score and oxygen saturation $\left(\mathrm{SpO}_{2}\right)$, we developed a better discriminating model for 1-year mortality, the DO index. The AUC value of the DO index was 0.73 for the prediction of 1-year mortality, and the AUC was 0.84 in the validated cohort, which was superior to the current palliative
Table 4. Survival analysis of 1-year mortality for different DO index scores of patients with severe and very severe COPD $(n=180)$.

\begin{tabular}{|c|c|c|c|c|c|}
\hline Score & Survived $(n)$ & Died $(n)$ & $\begin{array}{l}\text { Survival } \\
\text { rate (\%) }\end{array}$ & $\begin{array}{l}\text { Statistics } \\
\text { (chi-square) }\end{array}$ & $p$ Value $^{\mathrm{a}}$ \\
\hline DO & & & & 58.61 & $<0.001$ \\
\hline 2 & 56 & 2 & 96.55 & & \\
\hline 3 & 58 & 2 & 96.67 & & \\
\hline 4 & 30 & 3 & 90.91 & & \\
\hline 5 & 2 & 0 & 100.00 & & \\
\hline 8 & 1 & 0 & 100.00 & & \\
\hline 9 & 0 & 1 & 0 & & \\
\hline 10 & 10 & 2 & 83.3 & & \\
\hline 11 & 4 & 4 & 50 & & \\
\hline 12 & 1 & 2 & 33.3 & & \\
\hline 16 & 0 & 2 & 0 & & \\
\hline Score & Survived $(n)$ & Died $(n)$ & $\begin{array}{l}\text { Survival } \\
\text { rate (\%) }\end{array}$ & $\begin{array}{l}\text { Statistics } \\
\text { (chi-square) }\end{array}$ & $p$ Value $^{\mathrm{b}}$ \\
\hline DO & & & & 52.98 & $<0.001$ \\
\hline $2-7$ & 147 & 7 & 95.45 & & \\
\hline $8-11$ & 14 & 7 & 66.7 & & \\
\hline $12-16$ & 1 & 4 & 20.00 & & \\
\hline
\end{tabular}

guideline-recommended prediction tool. There was no process for sample size calculation in the study. Therefore, we calculated the statistical power backward using our sample size. According to the predictive ability of existing indices in a previous review ${ }^{23-25}$, we considered an AUC of 0.65 as the median discrimination power for previous predictors. Using our population of a total of 342 patients in the validation group for a two-sided $z$-test at a significance level of 0.05 , we achieved a $99 \%$ power to detect the AUC between the median discrimination power of previous predictors and the DO index in this study (PASS Power Analysis and Sample Size Software, NCSS, LLC., Kaysville, Utah). However, there were only 18 deaths out of 180 patients when the DO index was applied in severe COPD patients (see Table 4). The small numbers in some lattices may have thus affected the accuracy of the estimate. For example, when using a DO score $=9$ as a cutoff value, the 1-year survival rate for patients with DO scores $\geq 9$ was estimated to be $58 \%(15 / 26)$. The accuracy of the predictive ability, sensitivity, specificity, positive predictive value, and negative 
predictive value was $87.8 \% \quad(95 \% \mathrm{Cl}$ (confidence interval), $82.1-92.2 \%), \quad 61.1 \% \quad(95 \% \quad C l, 35.8-82.7), \quad 90.7 \% \quad(95 \% \quad C l$, $85.2-94.7), 42.3 \%(95 \% \mathrm{Cl}, 28.6-57.4)$, and $95.5 \%(95 \% \mathrm{Cl}$, 92.2-97.4), respectively. The low precision for sensitivity and the positive predictive value as indicated by wider confidence intervals was attributed to the smaller sample size in this test.

In general, the discriminative power of the model for the development group was better than that for the validation group. Patients in the validation group were older, had lower oxygenation, more comorbidities, more symptoms, and higher mortality than the development group in this study. The heterogeneity between our developmental and validation groups was one of the reasons explaining why the predictive ability was better in the validation group than in the development group. To evaluate whether the predictive ability of the DO index becomes stronger over time, we analyzed the predictive ability for 3- and 5-year mortality and found that the AUC values were 0.66 and 0.67 , respectively. These results implied that the DO index is not suitable for predicting 3- and 5-year mortality.

The findings of poor discrimination for the current models in this study were consistent with a previous systemic review conducted by Almagro et al. ${ }^{33}$. They used indicators already developed in previous articles to validate the performance in their cohort. Composite indices were better than a single parameter, and the best AUC was 0.68 for the CODEX index (comorbidity, obstruction, dyspnea, and previous exacerbation). The author concluded that no single index is good enough to guide the initiation of palliative care. Thus, the clinician should not make this decision based solely on a predictive tool. However, the use of the proposed DO index improved the predictive power $(A U C=0.73$ ) for 1-year mortality, and the AUC was 0.84 in the validated cohort. The BARC index for prognostic factors, including BMI and blood results (B), age (A), respiratory variables (airflow obstruction, exacerbations, smoking) (R), and comorbidities (C) was conducted based on medical databases and had a satisfactory AUC for 1-year mortality $(A U C=0.79)$. However, it included 18 variables in the model, such as age, BMI, FEV ${ }_{1}$, severe exacerbations, smoking status, multiple comorbidities, hemoglobin, platelets, and others for the evaluation ${ }^{34}$. In contrast, the DO index proposed in this study is simple to use because only two clinical parameters, dyspnea score and oxygenation, detected with a pulse oximeter, are needed. Another composite index, the ProPal-COPD tool, had a good predictive ability for 1-year mortality with an AUC of 0.82 . This model relied on the following seven predictors: (1) a surprise question, (2) MRC dyspnea, (3) the Clinical COPD Questionnaire (CCQ), (4) $\mathrm{FEV}_{1} \%$ of predicted value, (5) $\mathrm{BMI}$, (6) previous hospitalizations for AECOPD, and (7) specific comorbidities. However, some variables, such as the surprise question and $C C Q$, were not always routinely captured.

The DO index developed in this study was composed of the mMRC score and oxygen saturation. In a recent systematic review of predictive indicators in COPD, 24 models used composite indicators ${ }^{35}$. The dyspnea score (mMRC) is one of the ten most used parameters. Nishimura et al. also demonstrated that the severity of dyspnea was a more favorable predictor of death than $\mathrm{FEV}_{1}{ }^{17}$. This result was consistent with the use of $\mathrm{mMRC}$ in this article. Our model also included another indicator, $\mathrm{SpO}_{2}$, which was not commonly used in previous studies. Instead, some studies used arterial oxygen partial pressure $\left(\mathrm{PaO}_{2}\right)$ as a predictor. $\mathrm{PaO}_{2}$ is most often used during hospitalization, where $\mathrm{PaO}_{2}$ can fluctuate due to many factors, such as oxygen use, pneumonia, or cardiovascular instability. Additionally, drawing arterial blood may also lead to some local complications. In our study, we used $\mathrm{SpO}_{2}$ in stable patients breathing ambient air to measure constant oxygenation status. Lower levels of invasiveness are also preferred in outpatient settings.

The strength of this study is its diagnostic and measurement accuracy. COPD was diagnosed according to standard evaluations and spirometry results, and the deaths were verified by linking with a database from the Taiwan National Mortality Registry. In addition, we performed a sensitivity analysis by excluding a small amount of missing mMRC values and by restricting the subjects to patients with severe airflow limitations. Both results showed similar findings, which indicated the consistency of the discriminative validity. Finally, we validated the DO index and showed its good predictive ability for 1-year mortality in the second cohort of 342 patients.

There are limitations to our study. First, we did not have a large patient population and were only limited to one medical center. The second limitation of the present study was that few women (7.3\%) were enrolled. Recently, a large, real-world, cohort study revealed gender differences among COPD patients, where COPD was more frequent among women $(53.8 \%)$, and the overall mortality rate was higher in men as compared with women (45 vs. $38 \%$ ). However, no differences in mortality due to COPD related to gender were found ${ }^{36}$. The majority of participants with smokingrelated COPD in Taiwan are male ${ }^{37}$; the smoking prevalence for women is $<5 \%$ in Taiwan. In contrast to industrialized countries in the West, COPD morbidity remains male predominant in most Asian countries ${ }^{37-39}$. However, the result of this study cannot be directly generalizable to other countries due to this limitation.

The third limitation was that socioeconomic status (SES) was not included in our predictive model. SES disadvantages appear to have a significant impact on COPD mortality and morbidity, where individuals with the lowest SES consistently had been shown to have significantly higher mortality than those with the highest $\mathrm{SES}^{40,41}$. We thus suggest including an SES measurement in the predictive model in further studies.

In conclusion, this study demonstrated that the predictive values for 1-year mortality were poor, based on the current recommendations for palliative care among COPD patients, including four different composite indices. The newly developed DO index proposed in this work exhibited better predictive ability than other alternatives. We suggest that COPD patients with DO index scores $\geq 12$, for example, patients with $\mathrm{mMRC}=4$ and $\mathrm{SpO}_{2}$ $<90 \%$, are good candidates to receive palliative care.

\section{DATA AVAILABILITY}

Full data sets are not available publicly currently for protecting patient privacy. But the data can be requested reasonably to the corresponding author.

Received: 6 November 2020; Accepted: 25 November 2021; Published online: 13 January 2022

\section{REFERENCES}

1. Rabe, K. F. et al. Global strategy for the diagnosis, management, and prevention of chronic obstructive pulmonary disease: GOLD executive summary. Am. J. Respir. Crit. Care Med. 176, 532-555 (2007).

2. Tashkin, D. P. et al. A 4-year trial of tiotropium in chronic obstructive pulmonary disease. N. Engl. J. Med. 359, 1543-1554 (2008).

3. Yang, I. A., Fong, K. M., Sim, E. H., Black, P. N. \& Lasserson, T. J. Inhaled corticosteroids for stable chronic obstructive pulmonary disease. Cochrane Database Syst. Rev. CD002991 (2007).

4. Calverley, P. M. et al. Salmeterol and fluticasone propionate and survival in chronic obstructive pulmonary disease. N. Engl. J. Med. 356, 775-789 (2007).

5. Au, D. H., Udris, E. M., Fihn, S. D., McDonell, M. B. \& Curtis, J. R. Differences in health care utilization at the end of life among patients with chronic obstructive pulmonary disease and patients with lung cancer. Arch. Intern. Med. 166, 326-331 (2006).

6. Jabbarian, L. J. et al. Advance care planning for patients with chronic respiratory diseases: a systematic review of preferences and practices. Thorax 73, 222-230 (2018).

7. Higginson, I. J. et al. An integrated palliative and respiratory care service for patients with advanced disease and refractory breathlessness: a randomized controlled trial. Lancet Respir. Med. 2, 979-987 (2014). 
8. Crawford, A. Respiratory practitioners' experience of end-of-life discussions in COPD. Br. J. Nurs. 19, 1164-1169 (2010).

9. Gott, M. et al. Barriers to advance care planning in chronic obstructive pulmonary disease. Palliat. Med. 23, 642-648 (2009).

10. Scheerens, $C$. et al. "A palliative end-stage COPD patient does not exist": a qualitative study of barriers to and facilitators for early integration of palliative home care for end-stage COPD. NPJ Prim. Care Respir. Med. https://doi.org/10.1038/ s41533-018-0091-9 (2018).

11. Gaspar, C., Alfarroba, S., Telo, L., Gomes, C. \& Barbara, C. End-of-life care in COPD: a survey carried out with Portuguese pulmonologists. Rev. Port. Pneumol. 20, 123-130 (2014).

12. Smith, T. A. et al. Specialist respiratory physicians' attitudes to and practice of advance care planning in COPD. A pilot study. Respir. Med. 108, 935-939 (2014).

13. MacPherson, A., Walshe, C., O'Donnell, V. \& Vyas, A. The views of patients with severe chronic obstructive pulmonary disease on advance care planning: a qualitative study. Palliat. Med. 27, 265-272 (2013).

14. Janssen, D. J. A., Spruit, M. A., Schols, J. M. G. A. \& Wouters, E. F. M. A call for highquality advance care planning in outpatients with severe COPD or chronic heart failure. Chest 139, 1081-1088 (2011).

15. Dijk, W. D. et al. Multidimensional prognostic indices for use in COPD patient care. A systematic review. Respir. Res. https://doi.org/10.1186/1465-9921-12-151 (2011).

16. Man, S. F. et al. C-reactive protein and mortality in mild to moderate chronic obstructive pulmonary disease. Thorax 61, 849-853 (2006).

17. Nishimura, K., Izumi, T., Tsukino, M. \& Oga, T. Dyspnea is a better predictor of 5 -year survival than airway obstruction in patients with COPD. Chest 121, 1434-1440 (2002).

18. Casanova, C. et al. Differential effect of modified medical research council dyspnea, COPD assessment test, and clinical COPD questionnaire for symptoms evaluation within the new GOLD staging and mortality in COPD. Chest 148, 159-168 (2015).

19. Celli, B. R. Predictors of mortality in COPD. Respir. Med. 104, 773-779 (2010).

20. Celli, B. R. et al. The body-mass index, airflow obstruction, dyspnea, and exercise capacity index in chronic obstructive pulmonary disease. N. Engl. J. Med. 350, 1005-1012 (2004).

21. Vestbo, J. et al. Global strategy for the diagnosis, management, and prevention of chronic obstructive pulmonary disease: GOLD executive summary. Am. J. Respir. Crit. Care Med. 187, 347-365 (2013).

22. Smith, L. E. et al. Prognostic variables and scores identifying the end of life in COPD: a systematic review. Int. J. Chron. Obstruct. Pulmon. Dis. 12, 2239-2256 (2013).

23. Marin, J. M. et al. Multicomponent indices to predict survival in COPD: the COCOMICS study. Eur. Respir. J. 42, 323-332 (2013).

24. Boeck, L. et al. Prognostic assessment in COPD without lung function: the B-AE-D indices. Eur. Respir. J. 47, 1635-1644 (2016).

25. Bloom, C. I., Ricciardi, F., Smeeth, L., Stone, P. \& Quint, J. K. Predicting COPD 1-year mortality using prognostic predictors routinely measured in primary care. $B M C$ Med. https://doi.org/10.1186/s12916-019-1310-0 (2019).

26. Chen, C. Z. et al. Using post-bronchodilator FEV1 is better than prebronchodilator FEV1 in evaluation of COPD severity. COPD 9, 276-280 (2012).

27. Blonshine, S., Mottram, C. D. \& Wanger, J. Pulmonary Function Laboratory Management and Procedure Manual 2nd edn (American Thoracic Society, 2005).

28. Bestall, J. C. et al. Usefulness of the medical research council (MRC) dyspnea scale as a measure of disability in patients with chronic obstructive pulmonary disease. Thorax 54, 581-586 (1999).

29. Charlson, M. E., Pompei, P., Ales, K. L. \& MacKenzie, C. R. A new method of classifying prognostic comorbidity in longitudinal studies: development and validation. J. Chronic Dis. 40, 373-383 (1987).

30. Lanken, P. N. et al. ATS End-of-Life Care Task Force. An official American Thoracic Society clinical policy statement: palliative care for patients with respiratory diseases and critical illnesses. Am. J. Respir. Crit. Care Med. 177, 912-927 (2008).

31. O'Donnell, D. E. et al. Canadian Thoracic Society recommendations for management of chronic obstructive pulmonary disease - 2008 update. Can. Respir. J. 15, 1A-8A (2008).

32. Thomas, K., Wilson, J. A. \& GSF Team. GSF PIG 6th Edition. National Gold Standards Framework Centre in end of life care. http://www.goldstandardsframework. org.uk (2016)

33. Almagro, P. et al. Palliative care and prognosis in COPD: a systematic review with a validation cohort. Int. J. Chron. Obstruct. Pulmon. Dis. 12, 1721-1729 (2017)

34. Yohannes, A. M., Baldwin, R. C. \& Connolly, M. J. Predictors of 1-year mortality in patients discharged from hospital following acute exacerbation of chronic obstructive pulmonary disease. Age Ageing 34, 491-496 (2005).
35. Bellou, V., Belbasis, L., Konstantinidis, A. K., Tzoulaki, I. \& Evangelou, E. Prognostic models for outcome prediction in patients with chronic obstructive pulmonary disease: systematic review and critical appraisal. BMJ https://doi.org/10.1136/bmj. 15358 (2019).

36. Lisspers, K. et al. Gender differences among Swedish COPD patients: results from the ARCTIC, a real-world retrospective cohort study. NPJ Prim. Care Respir. Med. https://doi.org/10.1038/s41533-019-0157-3 (2019)

37. Jamrozik, E. \& Musk, A. W. Respiratory health issues in the Asia-Pacific region: an overview. Respirology 16, 3-12 (2011).

38. Tan, W. C. \& Ng, T. P. COPD in Asia: where East meets West. Chest 133, 517-527 (2008).

39. Tan, W. C. Trends in chronic obstructive pulmonary disease in the Asia-Pacific regions. Curr. Opin. Pulm. Med. 17, 56-61 (2011).

40. Gershon, A. S., Dolmage, T. E., Stephenson, A. \& Jackson, B. Chronic obstructive pulmonary disease and socioeconomic status: a systematic review. COPD 9, 216-226 (2012).

41. Sahni, S., Talwar, A., Khanijo, S. \& Talwar, A. Socioeconomic status and its relationship to chronic respiratory disease. Adv. Respir. Med. 85, 97-108 (2017).

\section{ACKNOWLEDGEMENTS}

The study was funded by grants from the Ministry of Science and Technology (MOST107-2627-M-006-007, MOST 109-2314-B-006-091, MOST 110-2314-B-006-099), and it was supported in part by the Higher Education Sprout Project, Ministry of Education to the Headquarters of University Advancement at National Cheng Kung University (NCKU). We are grateful to the Health and Welfare Data Science Center of the Ministry of Health and Welfare and the Center of hospice palliative shared care at National Cheng Kung University Hospital (NCKUH) for providing all the data sets, facilities, and linkage services that were required for this study. Parts of our results were submitted as an e-poster in the ATS 2020 Virtual content.

\section{AUTHOR CONTRIBUTIONS}

T.-R.H. and C.-Z.C. were involved in the concept development and study design. All authors contributed to the data retrieval and patient recruitment. S.-H.T. and C.-Y.S. conducted the analysis. Paper drafting was mainly accomplished by S.-H.T. and C.-Z.C. and all authors contributed to the revision and approval of the final manuscript.

\section{COMPETING INTERESTS}

The authors declare no competing interests.

\section{ADDITIONAL INFORMATION}

Supplementary information The online version contains supplementary material available at https://doi.org/10.1038/s41533-021-00263-7.

Correspondence and requests for materials should be addressed to Chiung-Zuei Chen.

Reprints and permission information is available at http://www.nature.com/ reprints

Publisher's note Springer Nature remains neutral with regard to jurisdictional claims in published maps and institutional affiliations.

Open Access This article is licensed under a Creative Commons Attribution 4.0 International License, which permits use, sharing, adaptation, distribution and reproduction in any medium or format, as long as you give appropriate credit to the original author(s) and the source, provide a link to the Creative Commons license, and indicate if changes were made. The images or other third party material in this article are included in the article's Creative Commons license, unless indicated otherwise in a credit line to the material. If material is not included in the article's Creative Commons license and your intended use is not permitted by statutory regulation or exceeds the permitted use, you will need to obtain permission directly from the copyright holder. To view a copy of this license, visit http://creativecommons. org/licenses/by/4.0/.

(c) The Author(s) 2022 